\title{
The Stellar Structure of the Orion Spiral Arm in Selected Directions. I. The Galactic Anticenter
}

\section{Teimuraz M. Kvernadze}

Abastumani Astrophysical Observatory, 383762 Abastumani, Republic of Georgia

\section{Introduction}

To investigate the spatial distribution of various stellar groups in the HertzsprungRussell diagram, it is necessary to have homogeneous observational data of MK spectral classification and photometry. This enables us to perform some statistical analyses with sufficient accuracy.

This paper reports $B V$ photographic photometry of about 700 stars complete to $m_{V}=12$, in the direction of galactic anticenter with $(l, b)=\left(169^{\circ},-12.4^{\circ}\right)$, $\left(178^{\circ},-2.4^{\circ}\right),\left(184^{\circ}, 8.9^{\circ}\right)$. For the MK spectral data we used the catalogue by Bartaya and Kharadze (1992), which contains spectral and luminosity classes of 6042 stars in Kapteyn Areas Nos. 44-67. The observational data for this catalogue were obtained using the Abastumani Astrophysical Observatory $70 \mathrm{~cm}$ meniscus telescope and an $8^{\circ} \mathrm{objective} \mathrm{prism} \mathrm{with} \mathrm{dispersion} 166 \mathrm{Amm}^{-1}$ near $\mathrm{H} \gamma$. The errors in classification are equal to \pm 0.6 spectral subclasses and \pm 0.5 luminosity classes, with a limiting magnitude of $m_{V}=12.5$.

\section{Observational Data}

The photometric observational material was obtained with the $70 \mathrm{~cm}$ meniscus telescope at Abastumani in 1987-1989. At least 3 plates were exposed in each colour. The plate-filter combinations and exposure times were as follows: B, Kodak IIa-O, Schott GG13, 5 m; V, ORWO WO-1, Schott GG11, $10 \mathrm{~m}$.

In an effort to define transformation equations between the instrumental and international UBV systems, several fields with open clusters containing photoelectric sequences were observed. The reduction coefficients and their standard errors were found to be (Kvernadze, 1993a):

$$
\begin{aligned}
& V-v=(0.027 \pm 0.013)-(0.079 \pm 0.017) \cdot(B-V) \\
& B-b=(-0.075 \pm 0.018)+(0.192 \pm 0.020) \cdot(B-V)
\end{aligned}
$$

The rms (pg-pe) residuals for the calibration stars were found to be \pm 0.05 mag for both $B$ and $V$. The observational data obtained proved to be useful for investigation of the photometric field error of the telescope (Kvernadze 1993b). It was found that systematic deviations may reach several tenths of magnitudes and be corrected with a mean error of 0.08 mag.

Photographic iris photometry of plates was performed with the ASKORECORD - ASKOIRIS, Carl Zeiss, JENA of the Abastumani Observatory. 
Digitized iris sessions and accurate positions were automatically prepared on perfo-tape during measurements and afterwards were recorded to an IBM PC for further processing. Characteristic curve calibrations were carried out using a third or fourth-order polynomial least-squares technique. For each star, the final photographic magnitude was taken to be the mean of the individual magnitudes weighted with the inverse variance of the corresponding calibration curve. Photoelectric $U B V$ sequences were selected from several sources (Nicolet 1978; Lasker et al. 1988; Kazanasmas et al. 1981). A selection was also made from photometric catalogues of the Astronomical Data Center at Zvenigorod (Russia) in 1988.

Kapteyn Areas Nos. 48 and 50 contain an insufficient number of sequence stars for calibration over a whole range of magnitudes. So, we used tie-in fields, particularly KA-49 and KA-51 respectively, from the same declination zone $\left(+30^{\circ}\right)$ as the previous two. The tie-in pairs were exposed sequentially with a time interval of about $40 \mathrm{~m}$ and equal zenith distances. Photographic processing was absolutely identical. The tie-in field sequences were calibrated together and, if systematic errors occurred between them, then all stars were shifted towards the best one.

\section{Interstellar Absorption}

For the determination of visual interstellar extinction, $A_{v}$, as a function of distance from the Sun, colour excesses $E_{B-V}$ were defined for an individual star. Intrinsic colours and absolute magnitudes are from Schmidt-Kaler (1982) with $R=3.0$. Plots of extinction vs. distance show that the interstellar absorption is distributed quite non-uniformly within each field; the scatter is great even for $\mathrm{r}<500 \mathrm{pc}$. However, the mean absorptions are not so high. For KA-48, KA-49 and KA-50, $A_{V}=0.99,0.82$ and $0.70 \mathrm{mag}$ respectively, for a distance of $\mathrm{r}=2$ kpc. Bigay and Garnier (1970) obtained $A_{V}=0.7 \mathrm{mag}$ at $2 \mathrm{kpc}$ for KA-49; their extinction diagram is similar to ours.

From the same plots, at least two different $A_{v}(r)$ mean relationships are evident: a) a strong increase of extinction up to $2-3$ mag within $500 \mathrm{pc}$; b) a slow increase of extinction up to $1-1.5 \mathrm{kpc}$ with $A_{V}=0.5-0.7 \mathrm{mag}$, with nearly constant extinction at greater distances, even to $5 \mathrm{kpc}(!)$. This effect is most evident for KA-48 and agrees well with extinction maps of Neckel and Klare (1980) for KA-49 as it lies near the boundaries of their fields $177 /-1.0$ and 176/-3.0.

\section{The Space Distribution of Stars}

For the analyses of stellar densities of various spectral and luminosity classes on the basis of our observational data, we decided to select the following stellar groups: spectral classes B, A, F, G \& $\mathrm{K}$ and luminosity classes III, IV \& V. A more detailed division is impossible due to poor statistics of our data in the direction of the galactic anticenter. For the same reason we processed all three fields together. Nevertheless, we had to exclude FIII, GIII and KV groups as these contained practically no stars. 
One of the most important characteristics for investigating the space distribution of stars is the data completeness sphere radius $r_{0}$. There are some statistical methods for determination of this value (Borzov 1975). Kharadze et al. (1987) describe an algorithm for estimating $r_{0}$, but the relatively small amount of data in our stellar groups makes it difficult to use. However, we constructed a stellar frequency distribution vs. distance from the Sun for intervals of $100 \mathrm{pc}$. Taking into account the suggestion that stars with not very high luminosity are more or less uniformly distributed within $500 \mathrm{pc}$, we took as a first approach of $r_{0}$, an upper distance limit, corresponding to the frequency maxima.

Table 1 shows the results. The $r_{0, K}$ are values are from Kharadze et al. (1987) using the MK spectral catalogue by Bartaya (1979) with a limiting magnitude of $m_{V}=11.5$. The $D_{0}\left(10^{-3} \mathrm{pc}^{-3}\right)$ values are the stellar densities within the data completeness sphere, calculated as $D_{0}=N / V\left(r_{0}\right)$, where $N$ is the star number and $V\left(r_{0}\right)$ is the sum of corresponding conical volumes. Note that the results for the B stars are very rough as it is difficult to assume their uniform distribution even within the Orion Spiral Arm.

Table 1. The data completeness sphere radius (pc) and stellar densities

\begin{tabular}{cccccrcrrr}
\hline LC & \multicolumn{3}{c}{ III } & \multicolumn{4}{c}{ IV } & \multicolumn{3}{c}{ V } \\
SP & $r_{0}$ & $r_{0, K}$ & $D_{0}$ & $r_{0}$ & $r_{0, K}$ & $D_{0}$ & $r_{0}$ & $r_{0, K}$ & $D_{0}$ \\
\hline B & - & - & - & 900 & - & 0.011 & 700 & - & 0.028 \\
A & 700 & 401 & 0.009 & 500 & 327 & 0.060 & 400 & 273 & 0.155 \\
F & - & 174 & - & 300 & 221 & 0.168 & 200 & 163 & 1.263 \\
G & 300 & 263 & 0.032 & 200 & 156 & 0.296 & 100 & 62 & 7.556 \\
K & 300 & 277 & 0.104 & 200 & 96 & 0.376 & - & 24 & - \\
\hline \hline
\end{tabular}

\section{References}

Bartaya R. A. 1979, Bull. Abast. Astrophys. Obs., N51, 3

Bartaya R. A., \& Kharadze E. K. 1992, Bull.Abast.Astrophys.Obs., N72,3

Bigay J. H., \& Garnier R. 1970, Astr. Ap. Suppl., 1, 1

Borzov G. G. 1975, in Isledovanie Ekstremalno Molodikh Zvesdnikh Komplesov (Tashkent, FAN), p. 127

Kazanasmas M. S., Zavershneva L. A., \& Tomak L. F. 1981, Atlas i Katalog Zvezdnikh Velichin Fotoelektricheskikh Standartov (Kiev, Naukova Dumka)

Kharadze E. K., Bartaya R. A., Vereshchagin S. V., Dluzhnevskaya O. B., Pavlovskaya E. D. \& Piskunov A. E. 1987, Astron. Zh., 64, 696

Kvernadze T. M. 1993a, Bull. Abast. Astrophys. Obs., N73, 109

Kvernadze T. M. 1993b, Bull. Abast. Astrophys. Obs., N73, 117

Lasker B. M., Sturch C. R., Lopez C. et al., 1988, Ap. J. Suppl., 68, 1

Neckel Th., \& Klare G. 1980, Astr. Ap. Suppl., 42, 251 
Nicolet B. 1978, Astr. Ap. Suppl., 34, 1

Schmidt-Kaler Th. 1982, in Astronomie und Astrophysik, ed. H.H. Vogt (LandoltBörnstein), Springer Verlag, 2, 1 